\title{
Solving Integral Equations by Common Fixed Point Theorems on Complex Partial $b$-Metric Spaces
}

\author{
Arul Joseph Gnanaprakasam, ${ }^{1}$ Salah Mahmoud Boulaaras $\mathbb{D}^{2,3}$ Gunaseelan Mani, ${ }^{4}$ \\ Mohamed Abdalla $\mathbb{D}^{5,6}$ and Asma Alharbi ${ }^{2}$ \\ ${ }^{1}$ Department of Mathematics, College of Engineering and Technology, Faculty of Engineering and Technology, SRM Institute of \\ Science and Technology, SRM Nagar, Kattankulathur 603203, Kanchipuram, Chennai, Tamil Nadu, India \\ ${ }^{2}$ Department of Mathematics, College of Sciences and Arts, ArRas, Qassim University, Saudi Arabia \\ ${ }^{3}$ Laboratory of Fundamental and Applied Mathematics of Oran (LMFAO), University of Oran 1, Oran, 31000 Oran, Algeria \\ ${ }^{4}$ Department of Mathematics, Sri Sankara Arts and Science College (Autonomous), Affiliated to Madras University, Enathur, \\ 631 561, Kanchipuram, Tamil Nadu, India \\ ${ }^{5}$ Department of Mathematics, Faculty of Science, King Khalid University, Abha 61471, Saudi Arabia \\ ${ }^{6}$ Mathematics Department, Faculty of Science, South Valley University, Qena 83523, Egypt
}

Correspondence should be addressed to Salah Mahmoud Boulaaras; s.boularas@qu.edu.sa

Received 9 May 2021; Accepted 9 June 2021; Published 23 June 2021

Academic Editor: Liliana Guran

Copyright (c) 2021 Arul Joseph Gnanaprakasam et al. This is an open access article distributed under the Creative Commons Attribution License, which permits unrestricted use, distribution, and reproduction in any medium, provided the original work is properly cited.

In this paper, we prove some common fixed point theorems for rational contraction mapping on complex partial $b$-metric space. The presented results generalize and expand some of the literature's well-known results. We also explore some of the applications of our key results.

\section{Introduction}

Introduced in 1989 by Bakhtin [1] and Czerwick [2], the concept of $b$-metric spaces provided a framework to extend the results already known in the classical setting of metric spaces. The concept of complex valued metric spaces was introduced in 2011 by Azam et al. [3] and given some common fixed point theorems under the condition of contraction. Rao et al. [4] introduced the definition of complex valued $b$ -metric spaces in 2013 and provided a scheme to expand the results, as well as proved a common fixed point theorem under contraction. In 2017, Dhivya and Marudai [5] introduced the concept of complex partial metric space and suggested a plan to expand the results, as well as proved common fixed point theorems under the rational expression contraction condition. Gunaseelan $[6,7]$ presented the concept of complex partial $b$-metric space in 2019 , as well as proved the fixed point theorem under the contractive condition. Many researchers have studied some intriguing concepts and applications and have shown significant results [7-23]. In this paper, we prove some common fixed point theorems for rational contraction mapping on complex partial $b$-metric space.

\section{Preliminaries}

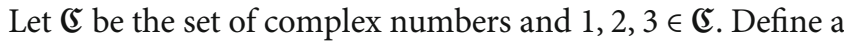
partial order $\leq$ on $\mathfrak{C}$ as follows: $1 \leq 2$ if and only if $\mathscr{R}(1) \leq$ $\mathscr{R}(2)$ and $\mathscr{I}(1) \leq \mathscr{I}(2)$.

Consequently, one can infer that $1 \leq 2$ if one of the following conditions is satisfied:

(i) $\mathscr{R}(1)=\mathscr{R}(2), \mathscr{I}(1)<\mathscr{I}(2)$

(ii) $\mathscr{R}(1)<\mathscr{R}(2), \mathscr{I}(1)=\mathscr{I}(2)$

(iii) $\mathscr{R}(1)<\mathscr{R}(2), \mathscr{I}(1)<\mathscr{I}(2)$

(iv) $\mathscr{R}(1)=\mathscr{R}(2), \mathscr{I}(1)=\mathscr{I}(2)$ 
In particular, we write $1 \precsim 2$ if $1 \neq 2$ and one of (i), (ii), and (iii) is satisfied and we write $1 \prec 2$ if only (iii) is satisfied. Notice that

(a) If $0 \leq 1 \precsim 2$, then $|1|<|2|$

(b) If $1 \leq 2$ and $2 \prec 3$, then $1 \prec 3$

(c) If $\eta, \gamma \in \mathbb{R}$ and $\eta \leq \gamma$, then $\eta_{1} \leq \gamma_{1}$ for all $0 \leq 1 \in \mathfrak{C}$

Here, $\mathfrak{c}_{+}\left(=\left\{(\aleph, \mathfrak{y}) \mid \aleph, \mathfrak{y} \geq 0, \aleph, \mathfrak{y} \in \mathbb{R}_{+}\right\}\right)$denote the set of nonnegative complex numbers.

Now, let us recall some basic concepts and notations, which will be used in the sequel.

Definition 1 [6]. A complex partial $b$-metric on a nonvoid set $\mathfrak{W}$ is a function $\varphi_{c b}: \mathfrak{W} \times \mathfrak{W} \longrightarrow \mathbb{C}_{+}$such that for all $\aleph, \vartheta$, $3 \in \mathfrak{W}$,

(i) $0 \leq \varphi_{c b}(\aleph, \aleph) \leq \varphi_{c b}(\aleph, \vartheta)($ small self-distances $)$

(ii) $\varphi_{c b}(\aleph, \vartheta)=\varphi_{c b}(\vartheta, \aleph)($ symmetry $)$

(iii) $\varphi_{c b}(\aleph, \aleph)=\varphi_{c b}(\aleph, \vartheta)=\varphi_{c b}(\vartheta, \vartheta) \Leftrightarrow \aleph=\vartheta($ equality $)$

(iv) $\exists$ a real number $s \geq 1$ and $s$ is an independent of $\aleph, \vartheta, \rightrightarrows$ such that $\varphi_{c b}(\aleph, \vartheta) \leq s\left[\varphi_{c b}(\aleph, \boldsymbol{Z})+\varphi_{c b}(\mathcal{Z}, \vartheta)\right]$ $-\varphi_{c b}(3,3)$ (triangularity)

A complex partial $b$-metric space is a pair $\left(\mathfrak{Z}, \varphi_{c b}\right)$ such that $\mathfrak{W}$ is a nonvoid set and $\varphi_{c b}$ is the complex partial $b$-metric on $\mathfrak{Z}$. The number $s$ is called the coefficient of $\left(\mathfrak{W}, \varphi_{c b}\right)$.

Definition 2 [6]. Let $\left(\mathfrak{W}, \varphi_{c b}\right)$ be a complex partial $b$-metric space with coefficient $s$. Let $\left\{\aleph_{n}\right\}$ be any sequence in $\mathfrak{Z}$ and $\aleph \in \mathfrak{W}$. Then,

(1) The sequence $\left\{\aleph_{n}\right\}$ is said to converge to $\aleph$, if $\lim _{n \rightarrow \infty} \varphi_{c b}\left(\aleph_{n}, \aleph\right)=\varphi_{c b}(\aleph, \aleph)$

(2) The sequence $\left\{\aleph_{n}\right\}$ is said to be Cauchy sequence in $\left(\mathfrak{W}, \varphi_{c b}\right)$ if $\lim _{n, m \rightarrow \infty} \varphi_{c b}\left(\aleph_{n}, \aleph_{m}\right)$ exists and is finite

(3) $\left(\mathfrak{W}, \varphi_{c b}\right)$ is said to be a complete complex partial $b$-metric space if for every Cauchy sequence $\left\{\aleph_{n}\right\}$ in $\mathfrak{Z}$, there exists $\aleph \in \mathfrak{Z}$ such that $\lim _{n, m \rightarrow \infty} \varphi_{c b}\left(\aleph_{n}\right.$, $\left.\aleph_{m}\right)=\lim _{n \longrightarrow \infty} \varphi_{c b}\left(\aleph_{n}, \aleph\right)=\varphi_{c b}(\aleph, \aleph)$.

Definition 3. Let $\mathfrak{S}$ and $\mathfrak{I}$ be self-mappings of nonempty set $\mathfrak{W}$. A point $\aleph \in \mathfrak{W}$ is called a common fixed point of $\mathfrak{S}$ and $\mathfrak{I}$ if $\aleph=\widetilde{S} \aleph=\mathfrak{I} \aleph$.

In 2019, Gunaseelan [6] proved some fixed point theorems on complex partial $b$-metric space as follows.

Theorem 4. Let $\left(\mathfrak{Z}, \varphi_{c b}\right)$ be any complete complex partial $b$ -metric space with coefficient $s \geq 1$ and $\mathfrak{S}: \mathfrak{W} \longrightarrow \mathfrak{W}$ be a mapping satisfying $\varphi_{c b}(\widetilde{\aleph}, \mathbb{\Im} \vartheta) \leq \lambda \max \left\{\varphi_{c b}(\aleph, \vartheta), \varphi_{c b}(\aleph, \widetilde{\aleph}), \varphi_{c b}(\vartheta, \widetilde{\vartheta})\right\}$

for all $\aleph, \vartheta \in \mathfrak{W}$, where $\lambda \in[0,1 / s]$. Then, $\mathfrak{\subseteq}$ has a unique fixed point $\aleph^{*} \in \mathfrak{W}$ and $\varphi_{c b}\left(\aleph^{*}, \aleph^{*}\right)=0$.

Inspired by the study made by Gunaseelan [6], here, we prove some common fixed point theorems for rational contraction mapping on complex partial $b$-metric space with an application.

\section{Main Results}

In this section, we will give our main result of this paper, where some common fixed point theorems for rational contraction mapping on complex partial $b$-metric space are given.

Theorem 5. Let $\left(\mathfrak{V B}, \varphi_{c b}\right)$ be a complete complex partial $b$-metric space with the coefficient $s \geq 1$ and $\mathfrak{S}, \mathfrak{T}: \mathfrak{W} \longrightarrow$ $\mathfrak{W}$ be mappings satisfying

$$
\varphi_{c b}(\mathfrak{S}, \mathfrak{I} \vartheta) \leq a_{1} \varphi_{c b}(\aleph, \vartheta)+\frac{a_{2} \varphi_{c b}(\aleph, \mathfrak{S} \aleph) \varphi_{c b}(\vartheta, \mathfrak{I} \vartheta)}{1+\varphi_{c b}(\aleph, \mathfrak{T} \vartheta)+\varphi_{c b}(\aleph, \vartheta)}
$$

for all $\aleph, \vartheta \in \mathfrak{W}$, where $a_{1}, a_{2}$ are nonnegative reals with $a_{1}+s a_{2}<1$. Then, $\mathfrak{S}$ and $\mathfrak{T}$ have a unique common fixed point in $\mathfrak{Z}$.

Proof. Let $\aleph_{0}$ be arbitrary point in $\mathfrak{W}$, and define a sequence $\left\{\aleph_{\mathfrak{n}}\right\}$ in $\mathfrak{B}$ such that

$$
\aleph_{2 \mathfrak{n}+1}=\mathfrak{S} \aleph_{2 \mathfrak{n}}, \aleph_{2 \mathfrak{n}+2}=\mathfrak{T} \aleph_{2 \mathfrak{n}+1}, \quad \forall \mathfrak{n} \geq 0
$$

Next, show that the sequence $\left\{\aleph_{n}\right\}$ is Cauchy. By using (3), we get

$$
\begin{aligned}
\varphi_{c b}\left(\aleph_{2 \mathfrak{n}+1}, \aleph_{2 \mathfrak{n}+2}\right)= & \varphi_{c b}\left(\mathfrak{S \aleph}_{2 \mathfrak{n}}, \mathfrak{T} \aleph_{2 \mathfrak{n}+1}\right) \\
\leq & \mathrm{a}_{1} \varphi_{c b}\left(\aleph_{2 \mathfrak{n}}, \aleph_{2 \mathfrak{n}+1}\right) \\
& +\frac{\mathrm{a}_{2} \varphi_{c b}\left(\aleph_{2 \mathfrak{n}}, \mathfrak{S} \aleph_{2 \mathfrak{n}}\right) \varphi_{c b}\left(\aleph_{2 \mathfrak{n}+1}, \mathfrak{T} \aleph_{2 \mathfrak{n}+1}\right)}{1+\varphi_{c b}\left(\aleph_{2 \mathfrak{n}}, \mathfrak{T} \aleph_{2 \mathfrak{n}+1}\right)+\varphi_{c b}\left(\aleph_{2 \mathfrak{n}}, \aleph_{2 \mathfrak{n}+1}\right)},
\end{aligned}
$$

so that

$$
\begin{aligned}
\left|\varphi_{c b}\left(\aleph_{2 \mathfrak{n}+1}, \aleph_{2 \mathfrak{n}+2}\right)\right| \leq & \mathrm{a}_{1}\left|\varphi_{c b}\left(\aleph_{2 \mathfrak{n}}, \aleph_{2 \mathfrak{n}+1}\right)\right| \\
& +\frac{\mathrm{a}_{2}\left|\varphi_{c b}\left(\aleph_{2 \mathfrak{n}}, \aleph_{2 \mathfrak{n}+1}\right)\right|\left|\varphi_{c b}\left(\aleph_{2 \mathfrak{n}+1}, \aleph_{2 \mathfrak{n}+2}\right)\right|}{\left|\varphi_{c b}\left(\aleph_{2 \mathfrak{n}}, \aleph_{2 \mathfrak{n}+2}\right)\right|+\left|\varphi_{c b}\left(\aleph_{2 \mathfrak{n}}, \aleph_{2 \mathfrak{n}+1}\right)\right|} .
\end{aligned}
$$


By the hypothesis of theorem, we get

$$
\begin{aligned}
\left|\varphi_{c b}\left(\aleph_{2 \mathfrak{n}+1}, \aleph_{2 \mathfrak{n}+2}\right)\right| \leq & s\left\{\left|\varphi_{c b}\left(\aleph_{2 \mathfrak{n}+1}, \aleph_{2 \mathfrak{n}}\right)\right|+\left|\varphi_{c b}\left(\aleph_{2 \mathfrak{n}}, \aleph_{2 \mathfrak{n}+2}\right)\right|\right\} \\
& -\left|\varphi_{c b}\left(\aleph_{2 \mathfrak{n}}, \aleph_{2 \mathfrak{n}}\right)\right|\left|\varphi_{c b}\left(\aleph_{2 \mathfrak{n}+1}, \aleph_{2 \mathfrak{n}+2}\right)\right| \\
\leq & s\left\{\left|\varphi_{c b}\left(\aleph_{2 \mathfrak{n}+1}, \aleph_{2 \mathfrak{n}}\right)\right|+\left|\varphi_{c b}\left(\aleph_{2 \mathfrak{n}}, \aleph_{2 \mathfrak{n}+2}\right)\right|\right\}
\end{aligned}
$$

Hence,

$$
\begin{aligned}
\left|\varphi_{c b}\left(\aleph_{2 \mathfrak{n}+1}, \aleph_{2 \mathfrak{n}+2}\right)\right| & \leq \mathrm{a}_{1}\left|\varphi_{c b}\left(\aleph_{2 \mathfrak{n}}, \aleph_{2 \mathfrak{n}+1}\right)\right|+s \mathrm{a}_{2}\left|\varphi_{c b}\left(\aleph_{2 \mathfrak{n}}, \aleph_{2 \mathfrak{n}+1}\right)\right| \\
& =\left(\mathrm{a}_{1}+s \mathrm{a}_{2}\right)\left|\varphi_{c b}\left(\aleph_{2 \mathfrak{n}}, \aleph_{2 \mathfrak{n}+1}\right)\right|\left|\varphi_{c b}\left(\aleph_{2 \mathfrak{n}+1}, \aleph_{2 \mathfrak{n}+2}\right)\right| \\
& \leq\left(\mathrm{a}_{1}+s \mathrm{a}_{2}\right)\left|\varphi_{c b}\left(\aleph_{2 \mathfrak{n}}, \aleph_{2 \mathfrak{n}+1}\right)\right| .
\end{aligned}
$$

Similarly,

$$
\left|\varphi_{c b}\left(\aleph_{2 \mathfrak{n}+2}, \aleph_{2 \mathfrak{n}+3}\right)\right| \leq\left(\mathrm{a}_{1}+s \mathrm{a}_{2}\right)\left|\varphi_{c b}\left(\aleph_{2 \mathfrak{n}+1}, \aleph_{2 \mathfrak{n}+2}\right)\right|,
$$

since $\mathrm{a}_{1}+s \mathrm{a}_{2}<1$. Therefore, with $\delta=\mathrm{a}_{1}+s \mathrm{a}_{2}<1$ and for all $\mathfrak{n} \geq 0$, consequently, we have

$$
\begin{aligned}
\left|\varphi_{c b}\left(\aleph_{2 \mathfrak{n}+1}, \aleph_{2 \mathfrak{n}+2}\right)\right| & \leq \delta\left|\varphi_{c b}\left(\aleph_{2 \mathfrak{n}}, \aleph_{2 \mathfrak{n}+1}\right)\right| \leq \delta^{2}\left|\varphi_{c b}\left(\aleph_{2 \mathfrak{n}-1}, \aleph_{2 \mathfrak{n}}\right)\right| \vdots \\
& \leq \delta^{2 \mathfrak{n}+1}\left|\varphi_{c b}\left(\aleph_{0}, \aleph_{1}\right)\right| .
\end{aligned}
$$

That is,

$$
\begin{aligned}
\left|\varphi_{c b}\left(\aleph_{\mathfrak{n}+1}, \aleph_{\mathfrak{n}+2}\right)\right| & \leq \delta\left|\varphi_{c b}\left(\aleph_{\mathfrak{n}}, \aleph_{\mathfrak{n}+1}\right)\right| \\
& \leq \delta^{2}\left|\varphi_{c b}\left(\aleph_{\mathfrak{n}-1}, \aleph_{\mathfrak{n}}\right)\right| \\
& \leq \delta^{\mathfrak{n}+1}\left|\varphi_{c b}\left(\aleph_{0}, \aleph_{1}\right)\right|
\end{aligned}
$$

For any $\mathfrak{m}>\mathfrak{n}, \mathfrak{m}, \mathfrak{n} \in \mathbb{N}$, we have

$$
\begin{aligned}
\left|\varphi_{c b}\left(\aleph_{\mathfrak{n}}, \aleph_{\mathfrak{m}}\right)\right| \leq & \left\{\left|\varphi_{c b}\left(\aleph_{\mathfrak{n}}, \aleph_{\mathfrak{n}+1}\right)\right|+\left|\varphi_{c b}\left(\aleph_{\mathfrak{n}+1}, \aleph_{\mathfrak{m}}\right)\right|\right\} \\
& -\left|\varphi_{c b}\left(\aleph_{\mathfrak{n}+1}, \aleph_{\mathfrak{n}+1}\right)\right| \leq s\left\{\left|\varphi_{c b}\left(\aleph_{\mathfrak{n}}, \aleph_{\mathfrak{n}+1}\right)\right|\right. \\
& \left.+\left|\varphi_{c b}\left(\aleph_{\mathfrak{n}+1}, \aleph_{\mathfrak{m}}\right)\right|\right\} \leq s\left|\varphi_{c b}\left(\aleph_{\mathfrak{n}}, \aleph_{\mathfrak{n}+1}\right)\right| \\
& +s^{2}\left|\varphi_{c b}\left(\aleph_{\mathfrak{n}+1}, \aleph_{\mathfrak{n}+2}\right)\right|+s^{2}\left|\varphi_{c b}\left(\aleph_{\mathfrak{n}+2}, \aleph_{\mathfrak{m}}\right)\right| \\
& -\left|\varphi_{c b}\left(\aleph_{\mathfrak{n}+2}, \aleph_{\mathfrak{n}+2}\right)\right| \leq s\left|\varphi_{c b}\left(\aleph_{\mathfrak{n}}, \aleph_{\mathfrak{n}+1}\right)\right| \\
& +s^{2}\left|\varphi_{c b}\left(\aleph_{\mathfrak{n}+1}, \aleph_{\mathfrak{n}+2}\right)\right|+s^{2}\left|\varphi_{c b}\left(\aleph_{\mathfrak{n}+2}, \aleph_{\mathfrak{m}}\right)\right| \\
\leq & s\left|\varphi_{c b}\left(\aleph_{\mathfrak{n}}, \aleph_{\mathfrak{n}+1}\right)\right|+s^{2}\left|\varphi_{c b}\left(\aleph_{\mathfrak{n}+1}, \aleph_{\mathfrak{n}+2}\right)\right| \\
& +s^{3}\left|\varphi_{c b}\left(\aleph_{\mathfrak{n}+2}, \aleph_{\mathfrak{n}+3}\right)\right|+s^{3}\left|\varphi_{c b}\left(\aleph_{\mathfrak{n}+3}, \aleph_{\mathfrak{m}}\right)\right| \\
& -\left|\varphi_{c b}\left(\aleph_{\mathfrak{n}+3}, \aleph_{\mathfrak{n}+3}\right)\right| \leq s\left|\varphi_{c b}\left(\aleph_{\mathfrak{n}}, \aleph_{\mathfrak{n}+1}\right)\right| \\
& +s^{2}\left|\varphi_{c b}\left(\aleph_{\mathfrak{n}+1}, \aleph_{\mathfrak{n}+2}\right)\right|+s^{3}\left|\varphi_{c b}\left(\aleph_{\mathfrak{n}+2}, \aleph_{\mathfrak{n}+3}\right)\right| \\
& +s^{3}\left|\varphi_{c b}\left(\aleph_{\mathfrak{n}+3}, \aleph_{\mathfrak{m}}\right)\right| \vdots \leq s\left|\varphi_{c b}\left(\aleph_{\mathfrak{n}}, \aleph_{\mathfrak{n}+1}\right)\right| \\
& +s^{2}\left|\varphi_{c b}\left(\aleph_{\mathfrak{n}+1}, \aleph_{\mathfrak{n}+2}\right)\right|+s^{3}\left|\varphi_{c b}\left(\aleph_{\mathfrak{n}+2}, \aleph_{\mathfrak{n}+3}\right)\right| \\
& +\cdots s^{\mathfrak{m}-\mathfrak{n}-2}\left|\varphi_{c b}\left(\aleph_{\mathfrak{m}-3}, \aleph_{\mathfrak{m}-2}\right)\right| \\
& +s^{\mathfrak{m}-\mathfrak{n}-1}\left|\varphi_{c b}\left(\aleph_{\mathfrak{m}-2}, \aleph_{\mathfrak{m}-1}\right)\right| \\
& +s^{\mathfrak{m}-\mathfrak{n}}\left|\varphi_{c b}\left(\aleph_{\mathfrak{m}-1}, \aleph_{\mathfrak{m}}\right)\right|
\end{aligned}
$$

From (10), we get

$$
\begin{aligned}
\left|\varphi_{c b}\left(\aleph_{\mathfrak{n}}, \aleph_{\mathfrak{m}}\right)\right| \leq & \delta^{\mathfrak{n}}\left|\varphi_{c b}\left(\aleph_{0}, \aleph_{1}\right)\right|+s^{2} \delta^{\mathfrak{n}+1}\left|\varphi_{c b}\left(\aleph_{0}, \aleph_{1}\right)\right| \\
& +s^{3} \delta^{\mathfrak{n}+2}\left|\varphi_{c b}\left(\aleph_{0}, \aleph_{1}\right)\right| \\
& +\cdots+s^{\mathfrak{m}-\mathfrak{n}-2} \delta^{\mathfrak{m}-3}\left|\varphi_{c b}\left(\aleph_{0}, \aleph_{1}\right)\right| \\
& +s^{\mathfrak{m}-\mathfrak{n}-1} \delta^{\mathfrak{m}-2}\left|\varphi_{c b}\left(\aleph_{0}, \aleph_{1}\right)\right| \\
& +s^{\mathfrak{m}-\mathfrak{n}} \delta^{\mathfrak{m}-1}\left|\varphi_{c b}\left(\aleph_{0}, \aleph_{1}\right)\right| \\
= & \sum_{i=1}^{\mathfrak{m}-\mathfrak{n}} s^{i} \delta^{i+\mathfrak{n}-1}\left|\varphi_{c b}\left(\aleph_{0}, \aleph_{1}\right)\right| .
\end{aligned}
$$

Hence,

$$
\begin{aligned}
\left|\varphi_{c b}\left(\aleph_{\mathfrak{n}}, \aleph_{\mathfrak{m}}\right)\right| & \leq \sum_{i=1}^{\mathfrak{m}-\mathfrak{n}} s^{i+\mathfrak{n}-1} \delta^{i+\mathfrak{n}-1}\left|\varphi_{c b}\left(\aleph_{0}, \aleph_{1}\right)\right| \\
& =\sum_{t=\mathfrak{n}}^{\mathfrak{m}-1} s^{t} \delta^{t}\left|\varphi_{c b}\left(\aleph_{0}, \aleph_{1}\right)\right| \leq \sum_{t=\mathfrak{n}}^{\infty}(s \delta)^{t}\left|\varphi_{c b}\left(\aleph_{0}, \aleph_{1}\right)\right| \\
& =\frac{(s \delta)^{\mathfrak{n}}}{1-s \delta}\left|\varphi_{c b}\left(\aleph_{0}, \aleph_{1}\right)\right|,
\end{aligned}
$$

and hence,

$\left|\varphi_{c b}\left(\aleph_{\mathfrak{n}}, \aleph_{\mathfrak{m}}\right)\right| \leq \frac{(s \delta)^{\mathfrak{n}}}{1-s \delta}\left|\varphi_{c b}\left(\aleph_{0}, \aleph_{1}\right)\right| \longrightarrow 0 \quad$ as $\mathfrak{m}, \mathfrak{n} \longrightarrow \infty$.

Thus, $\left\{\aleph_{\mathfrak{n}}\right\}$ is a Cauchy sequence in $\mathfrak{Z}$. Since $\mathfrak{Z}$ is complete, there exists some $\mathfrak{u} \in \mathfrak{V}$ such that $\aleph_{\mathfrak{n}} \longrightarrow \mathfrak{u}$ as $\mathfrak{n} \longrightarrow \infty$ and

$$
\varphi_{c b}(\mathfrak{u}, \mathfrak{u})=\lim _{n \longrightarrow \infty} \varphi_{c b}\left(\mathfrak{u}, \aleph_{\mathfrak{n}}\right)=\lim _{n \longrightarrow \infty} \varphi_{c b}\left(\aleph_{\mathfrak{n}}, \aleph_{\mathfrak{n}}\right)=0
$$

Assume on the contrary that there exists $z \in \mathfrak{W}$ such that

$$
\left|\varphi_{c b}(\mathfrak{u}, \mathfrak{S} \mathfrak{t})\right|=|\mathfrak{z}|>0
$$

By using the triangular inequality and (2), we obtain

$$
\begin{aligned}
& \mathfrak{z}=\varphi_{c b}(\mathfrak{u}, \widetilde{S} \mathfrak{u}) \\
& \leq s\left\{\varphi_{c b}\left(\mathfrak{u}, \aleph_{2 \mathfrak{n}+2}\right)+\varphi_{c b}\left(\aleph_{2 \mathfrak{n}+2}, \widetilde{S} \mathfrak{u}\right)\right\}-\varphi_{c b}\left(\aleph_{2 \mathfrak{n}+2}, \aleph_{2 \mathfrak{n}+2}\right) \\
& \leq s\left\{\varphi_{c b}\left(\mathfrak{u}, \aleph_{2 \mathfrak{n}+2}\right)+\varphi_{c b}\left(\aleph_{2 \mathfrak{n}+2}, \widetilde{S}_{\mathfrak{u}}\right)\right\} \\
& =s\left\{\varphi_{c b}\left(\mathfrak{u}, \aleph_{2 \mathfrak{n}+2}\right)+\varphi_{c b}\left(\mathfrak{T} \aleph_{2 \mathfrak{n}+1}, \mathfrak{S}_{\mathfrak{u}}\right)\right\} \\
& \leq s \varphi_{c b}\left(\mathfrak{u}, \aleph_{2 \mathfrak{n}+2}\right)+s \mathrm{a}_{1} \varphi_{c b}\left(\mathfrak{u}, \aleph_{2 \mathfrak{n}+1}\right) \\
& +\frac{s \mathrm{a}_{2} \varphi_{c b}(\mathfrak{u}, \mathfrak{S} \mathfrak{t}) \varphi_{c b}\left(\aleph_{2 \mathfrak{n}+1}, \mathfrak{\mathfrak { T }} \aleph_{2 \mathfrak{n}+1}\right)}{1+\varphi_{c b}\left(\mathfrak{u}, \mathfrak{T} \aleph_{2 \mathfrak{n}+1}\right)+\varphi_{c b}\left(\mathfrak{u}, \aleph_{2 \mathfrak{n}+1}\right)} \\
& =s \varphi_{c b}\left(\mathfrak{u}, \aleph_{2 \mathfrak{n}+2}\right)+s \mathrm{a}_{1} \varphi_{c b}\left(\mathfrak{u}, \aleph_{2 \mathfrak{n}+1}\right) \\
& +\frac{s \mathrm{a}_{2} \varphi_{c b}\left(\mathfrak{u}, \boldsymbol{S}_{\mathfrak{i}}\right) \varphi_{c b}\left(\aleph_{2 \mathfrak{n}+1}, \aleph_{2 \mathfrak{n}+2}\right)}{1+\varphi_{c b}\left(\mathfrak{u}, \aleph_{2 \mathfrak{n}+2}\right)+\varphi_{c b}\left(\mathfrak{u}, \aleph_{2 \mathfrak{n}+1}\right)},
\end{aligned}
$$


which implies that

$$
\begin{aligned}
|\mathfrak{z}|= & \left|\varphi_{c b}(\mathfrak{u}, \boldsymbol{S} \mathfrak{i})\right| \\
\leq & s\left|\varphi_{c b}\left(\mathfrak{u}, \aleph_{2 \mathfrak{n}+2}\right)\right|+s \mathrm{a}_{1}\left|\varphi_{c b}\left(\mathfrak{u}, \aleph_{2 \mathfrak{n}+1}\right)\right| \\
& +\frac{s \mathrm{a}_{2}\left|\varphi_{c b}\left(\mathfrak{u}, \boldsymbol{S}_{\mathfrak{u}}\right)\right|\left|\varphi_{c b}\left(\aleph_{2 \mathfrak{n}+1}, \aleph_{2 \mathfrak{n}+2}\right)\right|}{1+\varphi_{c b}\left(\mathfrak{u}, \aleph_{2 \mathfrak{n}+2}\right)|+| \varphi_{c b}\left(\mathfrak{u}, \aleph_{2 \mathfrak{n}+1}\right) \mid} .
\end{aligned}
$$

As $\mathfrak{n} \longrightarrow \infty$ in (18), we obtain that $|\mathfrak{z}|=\left|\varphi_{c b}(\mathfrak{t}, \mathfrak{S} \mathfrak{t})\right|$ $\leq 0$, a contradiction with (16). Therefore $|\mathfrak{z}|=0$. Hence, $\mathfrak{S}_{\mathfrak{t}}=\mathfrak{t}$. Similarly, we obtain $\mathfrak{T} \mathfrak{t}=\mathfrak{t}$.

Assume that $\mathfrak{u}^{*}$ is another common fixed point of $\mathfrak{S}$ and $\mathfrak{T}$. Then,

$$
\begin{aligned}
\varphi_{c b}\left(\mathfrak{u}, \mathfrak{t}^{*}\right) & =\varphi_{c b}\left(\mathfrak{S} \mathfrak{t}, \mathfrak{I}_{\mathfrak{t}^{*}}\right) \\
& \leq \mathrm{a}_{1} \varphi_{c b}\left(\mathfrak{u}, \mathfrak{u}^{*}\right)+\frac{\mathrm{a}_{2} \varphi_{c b}(\mathfrak{t}, \mathfrak{S} \mathfrak{t}) \varphi_{c b}\left(\mathfrak{t}^{*}, \mathfrak{I}_{\mathfrak{t}}^{*}\right)}{1+\varphi_{c b}\left(\mathfrak{u}, \mathfrak{I} \mathfrak{t} \mathfrak{t}^{*}\right)+\varphi_{c b}\left(\mathfrak{u}, \mathfrak{t}^{*}\right)},
\end{aligned}
$$

so that

$$
\begin{aligned}
\left|\varphi_{c b}\left(\mathfrak{u}, \mathfrak{t}^{*}\right)\right| \leq & \mathrm{a}_{1}\left|\varphi_{c b}\left(\mathfrak{u}, \mathfrak{t}^{*}\right)\right| \\
& +\frac{\mathrm{a}_{2}\left|\varphi_{c b}(\mathfrak{u}, \mathfrak{S} \mathfrak{t})\right|\left|\varphi_{c b}\left(\mathfrak{t}^{*}, \mathfrak{T}_{\mathfrak{t}}{ }^{*}\right)\right|}{1+\left|\varphi_{c b}\left(\mathfrak{u}, \mathfrak{\mathfrak { T }} \mathfrak{t}^{*}\right)\right|+\left|\varphi_{c b}\left(\mathfrak{u}, \mathfrak{t}^{*}\right)\right|} \\
\leq & \mathrm{a}_{1}\left|\varphi_{c b}\left(\mathfrak{u}, \mathfrak{t}^{*}\right)\right| .
\end{aligned}
$$

Hence, $\mathfrak{u}=\mathfrak{t}^{*}$, which proves the uniqueness. This completes the proof of the theorem.

Theorem 6. Let $\left(\mathfrak{W}, \varphi_{c b}\right)$ be a complete complex partial $b$-metric space with the coefficient $s \geq 1$ and $\mathfrak{S}, \mathfrak{I}: \mathfrak{W} \longrightarrow$ $\mathfrak{W}$ be mappings satisfying

$$
\begin{aligned}
\varphi_{c b}(\mathfrak{S}, \mathfrak{I} \vartheta) \leq & a_{1} \varphi_{c b}(\aleph, \vartheta)+a_{2} \varphi_{c b}(\aleph, \mathfrak{I} \vartheta) \\
& +a_{3}\left[\varphi_{c b}(\aleph, \mathfrak{S})+\varphi_{c b}(\vartheta, \mathfrak{I} \vartheta)\right]
\end{aligned}
$$

for all $\aleph, \vartheta \in \mathfrak{W}$, where $a_{1}, a_{2}$, and $a_{3}$ are nonnegative reals with $a_{1}+2 s a_{2}+2 a_{3}<1$. Then, $\mathfrak{S}$ and $\mathfrak{T}$ have a unique common fixed point in $\mathfrak{W}$.

Proof. Let $\aleph_{0}$ be arbitrary point in $\mathfrak{W}$, and define a sequence $\left\{\aleph_{\mathfrak{n}}\right\}$ in $\mathfrak{W}$ such that

$$
\aleph_{2 \mathfrak{n}+1}=\widetilde{S} \aleph_{2 \mathfrak{n}}, \aleph_{2 \mathfrak{n}+2}=\mathfrak{T} \aleph_{2 \mathfrak{n}+1}, \quad \forall \mathfrak{n} \geq 0
$$

Next, show that the sequence $\left\{\aleph_{\mathfrak{n}}\right\}$ is Cauchy. By using (22), we get

$$
\begin{aligned}
\varphi_{c b}\left(\aleph_{2 \mathfrak{n}+1}, \aleph_{2 \mathfrak{n}+2}\right)= & \varphi_{c b}\left(\mathfrak{S}_{2 \mathfrak{n}}, \mathfrak{T} \aleph_{2 \mathfrak{n}+1}\right) \\
\leq & \mathrm{a}_{1} \varphi_{c b}\left(\aleph_{2 \mathfrak{n}}, \aleph_{2 \mathfrak{n}+1}\right)+\mathrm{a}_{2} \varphi_{c b}\left(\aleph_{2 \mathfrak{n}}, \mathfrak{T} \aleph_{2 \mathfrak{n}+1}\right) \\
& +\mathrm{a}_{3}\left[\varphi_{c b}\left(\aleph_{2 \mathfrak{n}}, \mathfrak{S N}_{2 \mathfrak{n}}\right)+\varphi_{c b}\left(\aleph_{2 \mathfrak{n}+1}, \mathfrak{T} \aleph_{2 \mathfrak{n}+1}\right)\right] \\
= & \mathrm{a}_{1} \varphi_{c b}\left(\aleph_{2 \mathfrak{n}}, \aleph_{2 \mathfrak{n}+1}\right)+\mathrm{a}_{2} \varphi_{c b}\left(\aleph_{2 \mathfrak{n}}, \mathfrak{T} \aleph_{2 \mathfrak{n}+1}\right) \\
& +\mathrm{a}_{3}\left[\varphi_{c b}\left(\aleph_{2 \mathfrak{n}}, \aleph_{2 \mathfrak{n}+1}\right)+\varphi_{c b}\left(\aleph_{2 \mathfrak{n}+1}, \aleph_{2 \mathfrak{n}+2}\right)\right]
\end{aligned}
$$

so that

$$
\begin{aligned}
\left|\varphi_{c b}\left(\aleph_{2 \mathfrak{n}+1}, \aleph_{2 \mathfrak{n}+2}\right)\right| \leq & \mathrm{a}_{1}\left|\varphi_{c b}\left(\aleph_{2 \mathfrak{n}}, \aleph_{2 \mathfrak{n}+1}\right)\right| \\
& +\mathrm{a}_{2}\left|\varphi_{c b}\left(\aleph_{2 \mathfrak{n}}, \aleph_{2 \mathfrak{n}+2}\right)\right| \\
& +\mathrm{a}_{3}\left[\left|\varphi_{c b}\left(\aleph_{2 \mathfrak{n}}, \aleph_{2 \mathfrak{n}+1}\right)\right|\right. \\
& \left.+\left|\varphi_{c b}\left(\aleph_{2 \mathfrak{n}+1}, \aleph_{2 \mathfrak{n}+2}\right)\right|\right] .
\end{aligned}
$$

By the notion of complex partial $b$-metric space, we get

$$
\begin{aligned}
\left|\varphi_{c b}\left(\aleph_{2 \mathfrak{n}}, \aleph_{2 \mathfrak{n}+2}\right)\right| \leq & s\left\{\left|\varphi_{c b}\left(\aleph_{2 \mathfrak{n}}, \aleph_{2 \mathfrak{n}+1}\right)\right|+\left|\varphi_{c b}\left(\aleph_{2 \mathfrak{n}+1}, \aleph_{2 \mathfrak{n}+2}\right)\right|\right\} \\
& -\left|\varphi_{c b}\left(\aleph_{2 \mathfrak{n}+1}, \aleph_{2 \mathfrak{n}+1}\right)\right|\left|\varphi_{c b}\left(\aleph_{2 \mathfrak{n}}, \aleph_{2 \mathfrak{n}+2}\right)\right| \\
\leq & s\left\{\left|\varphi_{c b}\left(\aleph_{2 \mathfrak{n}}, \aleph_{2 \mathfrak{n}+1}\right)\right|+\left|\varphi_{c b}\left(\aleph_{2 \mathfrak{n}+1}, \aleph_{2 \mathfrak{n}+2}\right)\right|\right\} .
\end{aligned}
$$

Hence,

$$
\begin{aligned}
\left|\varphi_{c b}\left(\aleph_{2 \mathfrak{n}+1}, \aleph_{2 \mathfrak{n}+2}\right)\right| \leq & \mathrm{a}_{1}\left|\varphi_{c b}\left(\aleph_{2 \mathfrak{n}}, \aleph_{2 \mathfrak{n}+1}\right)\right| \\
& +s \mathrm{a}_{2}\left\{\left|\varphi_{c b}\left(\aleph_{2 \mathfrak{n}}, \aleph_{2 \mathfrak{n}+1}\right)\right|+\left|\varphi_{c b}\left(\aleph_{2 \mathfrak{n}+1}, \aleph_{2 \mathfrak{n}+2}\right)\right|\right\} \\
& +\mathrm{a}_{3}\left[\left|\varphi_{c b}\left(\aleph_{2 \mathfrak{n}}, \aleph_{2 \mathfrak{n}+1}\right)\right|+\left|\varphi_{c b}\left(\aleph_{2 \mathfrak{n}+1}, \aleph_{2 \mathfrak{n}+2}\right)\right|\right] \\
\leq & \left(\frac{\mathrm{a}_{1}+s \mathrm{a}_{2}+\mathrm{a}_{3}}{1-s \mathrm{a}_{2}-\mathrm{a}_{3}}\right)\left|\varphi_{c b}\left(\aleph_{2 \mathfrak{n}}, \aleph_{2 \mathfrak{n}+1}\right)\right| .
\end{aligned}
$$

Similarly,

$$
\left|\varphi_{c b}\left(\aleph_{2 \mathfrak{n}+2}, \aleph_{2 \mathfrak{n}+3}\right)\right| \leq\left(\frac{\mathrm{a}_{1}+s \mathrm{a}_{2}+\mathrm{a}_{3}}{1-s \mathrm{a}_{2}-\mathrm{a}_{3}}\right)\left|\varphi_{c b}\left(\aleph_{2 \mathfrak{n}+1}, \aleph_{2 \mathfrak{n}+2}\right)\right|
$$

Set $\delta=\left(\mathrm{a}_{1}+s \mathrm{a}_{2}+\mathrm{a}_{3}\right) /\left(1-s \mathrm{a}_{2}-\mathrm{a}_{3}\right)$. Since $\mathrm{a}_{1}+2 s \mathrm{a}_{2}+2$ $a_{3}<1$ and for all $\mathfrak{n} \geq 0$, consequently, we have

$$
\begin{aligned}
\left|\varphi_{c b}\left(\aleph_{2 \mathfrak{n}+1}, \aleph_{2 \mathfrak{n}+2}\right)\right| & \leq \delta\left|\varphi_{c b}\left(\aleph_{2 \mathfrak{n}}, \aleph_{2 \mathfrak{n}+1}\right)\right| \\
& \leq \delta^{2}\left|\varphi_{c b}\left(\aleph_{2 \mathfrak{n}-1}, \aleph_{2 \mathfrak{n}}\right)\right| \\
& \leq \delta^{2 \mathfrak{n}+1}\left|\varphi_{c b}\left(\aleph_{0}, \aleph_{1}\right)\right|
\end{aligned}
$$

That is,

$$
\begin{aligned}
\left|\varphi_{c b}\left(\aleph_{\mathfrak{n}+1}, \aleph_{\mathfrak{n}+2}\right)\right| & \leq \delta\left|\varphi_{c b}\left(\aleph_{\mathfrak{n}}, \aleph_{\mathfrak{n}+1}\right)\right| \\
& \leq \delta^{2}\left|\varphi_{c b}\left(\aleph_{\mathfrak{n}-1}, \aleph_{\mathfrak{n}}\right)\right| \\
& \leq \delta^{\mathfrak{n}+1}\left|\varphi_{c b}\left(\aleph_{0}, \aleph_{1}\right)\right| .
\end{aligned}
$$


For any $\mathfrak{m}>\mathfrak{n}, \mathfrak{m}, \mathfrak{n} \in \mathbb{N}$, we have

$$
\begin{aligned}
\left|\varphi_{c b}\left(\aleph_{\mathfrak{n}}, \aleph_{\mathfrak{m}}\right)\right| \leq & s\left\{\left|\varphi_{c b}\left(\aleph_{\mathfrak{n}}, \aleph_{\mathfrak{n}+1}\right)\right|+\left|\varphi_{c b}\left(\aleph_{\mathfrak{n}+1}, \aleph_{\mathfrak{m}}\right)\right|\right\} \\
& -\left|\varphi_{c b}\left(\aleph_{\mathfrak{n}+1}, \aleph_{\mathfrak{n}+1}\right)\right| \\
\leq & s\left\{\left|\varphi_{c b}\left(\aleph_{\mathfrak{n}}, \aleph_{\mathfrak{n}+1}\right)\right|+\left|\varphi_{c b}\left(\aleph_{\mathfrak{n}+1}, \aleph_{\mathfrak{m}}\right)\right|\right\} \\
\leq & s\left|\varphi_{c b}\left(\aleph_{\mathfrak{n}}, \aleph_{\mathfrak{n}+1}\right)\right|+s^{2}\left|\varphi_{c b}\left(\aleph_{\mathfrak{n}+1}, \aleph_{\mathfrak{n}+2}\right)\right| \\
& +s^{2}\left|\varphi_{c b}\left(\aleph_{\mathfrak{n}+2}, \aleph_{\mathfrak{m}}\right)\right|-\left|\varphi_{c b}\left(\aleph_{\mathfrak{n}+2}, \aleph_{\mathfrak{n}+2}\right)\right| \\
\leq & s\left|\varphi_{c b}\left(\aleph_{\mathfrak{n}}, \aleph_{\mathfrak{n}+1}\right)\right|+s^{2}\left|\varphi_{c b}\left(\aleph_{\mathfrak{n}+1}, \aleph_{\mathfrak{n}+2}\right)\right| \\
& +s^{2}\left|\varphi_{c b}\left(\aleph_{\mathfrak{n}+2}, \aleph_{\mathfrak{m}}\right)\right| \\
\leq & s\left|\varphi_{c b}\left(\aleph_{\mathfrak{n}}, \aleph_{\mathfrak{n}+1}\right)\right|+s^{2}\left|\varphi_{c b}\left(\aleph_{\mathfrak{n}+1}, \aleph_{\mathfrak{n}+2}\right)\right| \\
& +s^{3}\left|\varphi_{c b}\left(\aleph_{\mathfrak{n}+2}, \aleph_{\mathfrak{n}+3}\right)\right|+s^{3}\left|\varphi_{c b}\left(\aleph_{\mathfrak{n}+3}, \aleph_{\mathfrak{m}}\right)\right| \\
& -\left|\varphi_{c b}\left(\aleph_{\mathfrak{n}+3}, \aleph_{\mathfrak{n}+3}\right)\right| \\
\leq & s\left|\varphi_{c b}\left(\aleph_{\mathfrak{n}}, \aleph_{\mathfrak{n}+1}\right)\right|+s^{2}\left|\varphi_{c b}\left(\aleph_{\mathfrak{n}+1}, \aleph_{\mathfrak{n}+2}\right)\right| \\
& +s^{3}\left|\varphi_{c b}\left(\aleph_{\mathfrak{n}+2}, \aleph_{\mathfrak{n}+3}\right)\right|+s^{3}\left|\varphi_{c b}\left(\aleph_{\mathfrak{n}+3}, \aleph_{\mathfrak{m}}\right)\right| \\
\leq & s\left|\varphi_{c b}\left(\aleph_{\mathfrak{n}}, \aleph_{\mathfrak{n}+1}\right)\right|+s^{2}\left|\varphi_{c b}\left(\aleph_{\mathfrak{n}+1}, \aleph_{\mathfrak{n}+2}\right)\right| \\
& +s^{3}\left|\varphi_{c b}\left(\aleph_{\mathfrak{n}+2}, \aleph_{\mathfrak{n}+3}\right)\right| \\
& +\cdots s^{\mathfrak{m}-\mathfrak{n}-2}\left|\varphi_{c b}\left(\aleph_{\mathfrak{m}-3}, \aleph_{\mathfrak{m}-2}\right)\right| \\
& +s^{\mathfrak{m}-\mathfrak{n}-1}\left|\varphi_{c b}\left(\aleph_{\mathfrak{m}-2}, \aleph_{\mathfrak{m}-1}\right)\right| \\
& +s^{\mathfrak{m}-\mathfrak{n}}\left|\varphi_{c b}\left(\aleph_{\mathfrak{m}-1}, \aleph_{\mathfrak{m}}\right)\right|
\end{aligned}
$$

From (29), we get

$$
\begin{aligned}
\left|\varphi_{c b}\left(\aleph_{\mathfrak{n}}, \aleph_{\mathfrak{m}}\right)\right| \leq & s \delta^{\mathfrak{n}}\left|\varphi_{c b}\left(\aleph_{0}, \aleph_{1}\right)\right|+s^{2} \delta^{\mathfrak{n}+1}\left|\varphi_{c b}\left(\aleph_{0}, \aleph_{1}\right)\right| \\
& +s^{3} \delta^{\mathfrak{n}+2}\left|\varphi_{c b}\left(\aleph_{0}, \aleph_{1}\right)\right|+\cdots+s^{\mathfrak{m}-\mathfrak{n}-2} \delta^{\mathfrak{m}-3}\left|\varphi_{c b}\left(\aleph_{0}, \aleph_{1}\right)\right| \\
& +s^{\mathfrak{m}-\mathfrak{n}-1} \delta^{\mathfrak{m}-2}\left|\varphi_{c b}\left(\aleph_{0}, \aleph_{1}\right)\right|+s^{\mathfrak{m}-\mathfrak{n}} \delta^{\mathfrak{m}-1}\left|\varphi_{c b}\left(\aleph_{0}, \aleph_{1}\right)\right| \\
= & \sum_{i=1}^{\mathfrak{m}-\mathfrak{n}} s^{i} \delta^{i+\mathfrak{n}-1}\left|\varphi_{c b}\left(\aleph_{0}, \aleph_{1}\right)\right| .
\end{aligned}
$$

Hence,

$$
\begin{aligned}
\left|\varphi_{c b}\left(\aleph_{\mathfrak{n}}, \aleph_{\mathfrak{m}}\right)\right| & \leq \sum_{i=1}^{\mathfrak{m}-\mathfrak{n}} s^{i+\mathfrak{n}-1} \delta^{i+\mathfrak{n}-1}\left|\varphi_{c b}\left(\aleph_{0}, \aleph_{1}\right)\right| \\
& =\sum_{t=\mathfrak{n}}^{\mathfrak{m}-1} s^{t} \delta^{t}\left|\varphi_{c b}\left(\aleph_{0}, \aleph_{1}\right)\right| \\
& \leq \sum_{t=\mathfrak{n}}^{\infty}(s \delta)^{t}\left|\varphi_{c b}\left(\aleph_{0}, \aleph_{1}\right)\right| \\
& =\frac{(s \delta)^{\mathfrak{n}}}{1-s \delta}\left|\varphi_{c b}\left(\aleph_{0}, \aleph_{1}\right)\right|
\end{aligned}
$$

and hence,

$\left|\varphi_{c b}\left(\aleph_{\mathfrak{n}}, \aleph_{\mathfrak{m}}\right)\right| \leq \frac{(s \delta)^{\mathfrak{n}}}{1-s \delta}\left|\varphi_{c b}\left(\aleph_{0}, \aleph_{1}\right)\right| \longrightarrow 0 \quad$ as $\mathfrak{m}, \mathfrak{n} \longrightarrow \infty$

Thus, $\left\{\aleph_{\mathfrak{n}}\right\}$ is a Cauchy sequence in $\mathfrak{W}$. Since $\mathfrak{W}$ is complete, there exists some $\mathfrak{i t} \in \mathfrak{W}$ such that $\aleph_{\mathfrak{n}} \longrightarrow \mathfrak{t}$ as $\mathfrak{n}$ $\longrightarrow \infty$ and

Table 1

$$
\begin{array}{lc}
\hline(\aleph, \vartheta) & \varphi_{c b}(\aleph, \vartheta) \\
(1,1),(2,2) & 0 \\
(1,2),(2,1),(1,3),(3,1),(2,3),(3,2),(3,3) & e^{2 i x} \\
(1,4),(4,1),(2,4),(4,2),(3,4),(4,3),(4,4) & 9 e^{2 i x} \\
\hline & \\
\varphi_{c b}(\mathfrak{u}, \mathfrak{u})=\lim _{n \longrightarrow \infty} \varphi_{c b}\left(\mathfrak{u}, \aleph_{\mathfrak{n}}\right)=\lim _{n \longrightarrow \infty} \varphi_{c b}\left(\aleph_{\mathfrak{n}}, \aleph_{\mathfrak{n}}\right)=0 .
\end{array}
$$

Assume on the contrary that there exists $z \in \mathfrak{W}$ such that

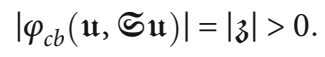

By using the triangular inequality and (21), we obtain

$$
\begin{aligned}
& \mathfrak{z}=\varphi_{c b}(\mathfrak{u}, \widetilde{\mathfrak{w}}) \\
& \leq s\left\{\varphi_{c b}\left(\mathfrak{u}, \aleph_{2 \mathfrak{n}+2}\right)+\varphi_{c b}\left(\aleph_{2 \mathfrak{n}+2}, \boldsymbol{\Phi} \mathfrak{t}\right)\right\}-\varphi_{c b}\left(\aleph_{2 \mathfrak{n}+2}, \aleph_{2 \mathfrak{n}+2}\right) \\
& \leq s\left\{\varphi_{c b}\left(\mathfrak{u}, \aleph_{2 \mathfrak{n}+2}\right)+\varphi_{c b}\left(\aleph_{2 \mathfrak{n}+2}, \widetilde{S} \mathfrak{u}\right)\right\} \\
& =s\left\{\varphi_{c b}\left(\mathfrak{u}, \aleph_{2 \mathfrak{n}+2}\right)+\varphi_{c b}\left(\mathfrak{S} \mathfrak{t}, \mathfrak{T} \mathfrak{\aleph}_{2 \mathfrak{n}+1}\right)\right\} \\
& \leq s \varphi_{c b}\left(\mathfrak{u}, \aleph_{2 \mathfrak{n}+2}\right)+s \mathrm{a}_{1} \varphi_{c b}\left(\mathfrak{u}, \aleph_{2 \mathfrak{n}+1}\right)+s \mathrm{a}_{2} \varphi_{c b}\left(\mathfrak{u}, \mathfrak{T} \aleph_{2 \mathfrak{n}+1}\right) \\
& +s \mathrm{a}_{3}\left[\varphi_{c b}(\mathfrak{u}, \widetilde{S} \mathfrak{t})+\varphi_{c b}\left(\aleph_{2 \mathfrak{n}+1}, \mathfrak{T} \aleph_{2 \mathfrak{n}+1}\right)\right] \\
& =s \varphi_{c b}\left(\mathfrak{u}, \aleph_{2 \mathfrak{n}+2}\right)+s \mathrm{a}_{1} \varphi_{c b}\left(\mathfrak{u}, \aleph_{2 \mathfrak{n}+1}\right)+s \mathrm{a}_{2} \varphi_{c b}\left(\mathfrak{u}, \aleph_{2 \mathfrak{n}+2}\right) \\
& +s \mathrm{a}_{3}\left[\mathfrak{z}+\varphi_{c b}\left(\aleph_{2 \mathfrak{n}+1}, \aleph_{2 \mathfrak{n}+2}\right)\right] \text {, }
\end{aligned}
$$

which implies that

$$
\begin{aligned}
|\mathfrak{z}|= & \left|\varphi_{c b}(\mathfrak{u}, \widetilde{S} \mathfrak{t})\right| \\
\leq & s\left|\varphi_{c b}\left(\mathfrak{u}, \aleph_{2 \mathfrak{n}+2}\right)\right|+s \mathrm{a}_{1}\left|\varphi_{c b}\left(\mathfrak{u}, \aleph_{2 \mathfrak{n}+1}\right)\right| \\
& +s \mathrm{a}_{2}\left|\varphi_{c b}\left(\mathfrak{u}, \aleph_{2 \mathfrak{n}+2}\right)\right|+s \mathrm{a}_{3}\left[|\mathfrak{z}|+\left|\varphi_{c b}\left(\aleph_{2 \mathfrak{n}+1}, \aleph_{2 \mathfrak{n}+2}\right)\right|\right]
\end{aligned}
$$

As $\mathfrak{n} \longrightarrow \infty$ in (37), we obtain that $|\mathfrak{z}|=\mid \varphi_{c b}(\mathfrak{t}, \mathfrak{S} \mathfrak{t})$ $\mid \leq 0$, a contradiction with (35). Therefore, $|z|=0$. Hence, $\widetilde{S} \mathfrak{t}=\mathfrak{u}$. Similarly, we obtain $\mathfrak{T} \mathfrak{t}=\mathfrak{t}$.

Assume that $\mathfrak{u}^{*}$ is another common fixed point of $\mathfrak{S}$ and $\mathfrak{T}$. Then,

$$
\begin{aligned}
\varphi_{c b}\left(\mathfrak{u}, \mathfrak{t}^{*}\right)= & \varphi_{c b}\left(\widetilde{S} \mathfrak{t}, \mathfrak{T}_{\mathfrak{t}}{ }^{*}\right) \\
\leq & \mathrm{a}_{1} \varphi_{c b}\left(\mathfrak{u}, \mathfrak{u}^{*}\right)+\mathrm{a}_{2} \varphi_{c b}\left(\mathfrak{u}, \mathfrak{I}_{\mathfrak{t}}{ }^{*}\right) \\
& +\mathrm{a}_{3}\left[\varphi_{c b}(\mathfrak{u}, \mathfrak{S} \mathfrak{t})+\varphi_{c b}\left(\mathfrak{u}^{*}, \mathfrak{T}_{\mathfrak{u}}{ }^{*}\right)\right],
\end{aligned}
$$

which implies that $\left|\varphi_{c b}\left(\mathfrak{u}, \mathfrak{t}^{*}\right)\right| \leq 0$, a contradiction. So $\mathfrak{t}=$ $\mathfrak{t}^{*}$, which proves the uniqueness.

Example 1. Let $\mathfrak{W}=\{1,2,3,4\}$ be endowed with the order $\aleph \leq \vartheta$ if and only if $\vartheta \leq \aleph$. Then, $\leq$ is a partial order in $\mathfrak{W}$. Define the complex partial $b$-metric space $\varphi_{c b}: \mathfrak{W} \times \mathfrak{W}$ $\longrightarrow \mathbb{C}_{+}$as follows (Table 1): 
It is easy to verify that $\left(\mathfrak{W}, \varphi_{c b}\right)$ is a complete complex partial $b$-metric space with the coefficient $s \geq 1$ for $x \in[0, \pi / 2]$. Define $\mathfrak{S}, \mathfrak{I}: \mathfrak{W} \longrightarrow \mathfrak{W}$ by $\mathfrak{S} \mathfrak{N}=1$,

$$
\mathfrak{I}(\aleph)=\left(\begin{array}{ll}
1 & \text { if } \aleph \in\{1,2,3\}, \\
2 & \text { if } \aleph=4 .
\end{array}\right.
$$

Let $\lambda_{1}=1 / 9$ and $\lambda_{2}=1 / 8$; we consider the following cases:

(1) If $\aleph=1$ and $\vartheta \in \mathfrak{W}-\{4\}$, then $\mathfrak{S}(\aleph)=\mathfrak{T}(\vartheta)=1$ and the conditions of Theorem 5 are satisfied

(2) If $\aleph=1$ and $\vartheta=4$, then $\mathfrak{S} \mathcal{N}=1, \mathfrak{T} \vartheta=2$,

$$
\begin{aligned}
\varphi_{c b}(\mathfrak{S}, \mathfrak{I} \vartheta) & =e^{2 i x} \leq 9 \lambda_{1} e^{i 2 x} \\
& =\lambda_{1} \varphi_{c b}(\aleph, \vartheta)+\lambda_{2} \frac{\varphi_{c b}(\aleph, \mathfrak{S} \aleph) \varphi_{c b}(\vartheta, \mathfrak{I} \vartheta)}{1+\varphi_{c b}(\aleph, \mathfrak{I} \vartheta)+\varphi_{c b}(\aleph, \vartheta)}
\end{aligned}
$$

(3) If $\aleph=2$ and $\vartheta=4$, then $\mathfrak{S} \mathcal{N}=1, \mathfrak{T} \vartheta=2$

$$
\begin{aligned}
\varphi_{c b}(\mathfrak{S}, \mathfrak{T} \vartheta) & =e^{2 i x} \leq\left(1+\frac{1}{8}\right) e^{i 2 x} \\
& =\lambda_{1} 9 e^{i 2 x}+\lambda_{2} \frac{e^{i 2 x} 9 e^{i 2 x}}{1+9 e^{i 2 x}} \\
& =\lambda_{1} \varphi_{c b}(\aleph, \vartheta)+\lambda_{2} \frac{\varphi_{c b}(\aleph, \mathfrak{S} \aleph) \varphi_{c b}(\vartheta, \mathfrak{I} \vartheta}{1+\varphi_{c b}(\aleph, \mathfrak{I} \vartheta)+\varphi_{c b}(\aleph, \vartheta)}
\end{aligned}
$$

(4) If $\aleph=3$ and $\vartheta=4$, then $\mathfrak{S} \aleph=1, \mathfrak{T} \vartheta=2$

$$
\begin{aligned}
\varphi_{c b}(\mathfrak{S}, \mathfrak{I} \vartheta) & =e^{2 i x} \leq\left(1+\frac{9}{80}\right) e^{i 2 x} \\
& =\lambda_{1} 9 e^{i 2 x}+\lambda_{2} \frac{e^{i 2 x} 9 e^{i 2 x}}{1+10 e^{i 2 x}} \\
& =\lambda_{1} \varphi_{c b}(\aleph, \vartheta)+\lambda_{2} \frac{\varphi_{c b}(\aleph, \mathfrak{S} \aleph) \varphi_{c b}(\mathfrak{\vartheta}, \mathfrak{T} \vartheta)}{1+\varphi_{c b}(\aleph, \mathfrak{I} \vartheta)+\varphi_{c b}(\aleph, \vartheta)}
\end{aligned}
$$

(5) If $\aleph=4$ and $\vartheta=4$, then $\mathfrak{S} \aleph=1, \mathfrak{T} \vartheta=2$

$$
\begin{aligned}
\varphi_{c b}(\mathbb{S}, \mathfrak{T} 9) & =e^{2 i x} \leq\left(1+\frac{9}{16}\right) e^{i 2 x} \\
& =\lambda_{1} 9 e^{i 2 x}+\lambda_{2} \frac{9 e^{i 2 x} 9 e^{i 2 x}}{1+9 e^{i 2 x}+9 e^{i 2 x}} \\
& =\lambda_{1} \varphi_{c b}(\aleph, \vartheta)+\lambda_{2} \frac{\varphi_{c b}(\mathcal{N}, \mathfrak{S}) \varphi_{c b}(\vartheta, \mathfrak{I} \vartheta)}{1+\varphi_{c b}(\aleph, \mathfrak{I} \vartheta)+\varphi_{c b}(\aleph, \vartheta)}
\end{aligned}
$$

Moreover for $\mathrm{a}_{1}+s \mathrm{a}_{2}<1$, the conditions of Theorem 5 are satisfied. Therefore, 1 is the unique common fixed point of $\mathfrak{S}$ and $\mathfrak{T}$.

\section{Application}

Consider the following systems of integral equations:

$$
\begin{aligned}
& w(s)=b(s)+\int_{a}^{b} T_{1}(s, p, w(p)) d p, \\
& w(s)=b(s)+\int_{a}^{b} T_{2}(s, p, w(p)) d p,
\end{aligned}
$$

where

(1) $[$ label $=()]$

(2) $w(s)$ is an unknown variable for each $s \in J=[a, b], b$ $>a \geq 0$

(3) $b(s)$ is the deterministic free term defined for $s \in[a, b]$

(4) $T_{1}(s, p)$ and $T_{2}(s, p)$ are deterministic kernels defined for $s, p \in J=[a, b]$

In this section, we present an existence theorem for a common solution to (44) and (45) that belongs to $\mathfrak{W}=$ $\left(C(J), \mathbb{R}^{n}\right)$ (the set of continuous functions defined on $J$ ) by using the obtained result in Theorem 5 . We consider the continuous mappings $\mathfrak{S}, \mathfrak{I}: \mathfrak{W} \longrightarrow \mathfrak{W}$ given by

$$
\begin{array}{ll}
\mathfrak{S} w(s)=b(s)+\int_{a}^{b} T_{1}(s, p, w(p)) d p, & w \in \mathfrak{W}, s \in J, \\
\mathfrak{T} w(s)=b(s)+\int_{a}^{b} T_{2}(s, p, w(p)) d p, & z \in \mathfrak{W}, s \in J .
\end{array}
$$

Then, the existence of a common solution to the integral equations (44) and (45) is equivalent to the existence of a common fixed point of $T_{1}$ and $T_{2}$. It is well known that $\mathfrak{W}$, endowed with the metric $\varphi_{c b}$ defined by

$$
\varphi_{c b}(w, z)=|w(s)-z(s)|^{2}+2,
$$

for all $w, z \in \mathfrak{B}$, is a complete complex partial $b$-metric space. $\mathfrak{W}$ can also be equipped with the partial order $\leq$ given by

$w, z \in \mathfrak{W}, \quad w \leq z$ if and only $w(s) \geq z(s)$, for all $s \in J$. 
Further, let us consider that a system of integral equation as (44) and (45) under the following condition holds:

(1) $T_{1}, T_{2}: J \times J \times \mathbb{R}^{n} \longrightarrow \mathbb{R}^{n}$ are continuous functions satisfying

$$
\left|T_{1}(s, p, w(p))-T_{2}(s, p, z(p))\right| \leq \sqrt{\frac{\varphi_{c b}(w, z)}{(b-a) e^{t}}-\frac{2}{b-a}},
$$

Theorem 7. Let $\left(C(J), \mathbb{R}^{n}, \varphi_{c b}\right)$ be a complete complex partial $b$-metric space; then, the systems (44) and (45) under condition (3) have a unique common solution.

Proof. For $w, z \in\left(C(J), \mathbb{R}^{n}\right)$ and $s \in J$, we define the continuous mappings $\mathfrak{S}, \mathfrak{I}: \mathfrak{W} \longrightarrow \mathfrak{W}$ by

$$
\begin{aligned}
& \mathfrak{S} w(s)=b(s)+\int_{a}^{b} T_{1}(s, p, w(p)) d p \\
& \mathfrak{T} w(s)=b(s)+\int_{a}^{b} T_{2}(s, p, w(p)) d p .
\end{aligned}
$$

Then, we have

$$
\begin{aligned}
\varphi_{c b}(\mathfrak{\Im} w(s), \mathfrak{I} z(s)) & =|\mathfrak{S} w(s)-\mathfrak{\mathfrak { T }} z(s)|^{2}+2 \\
& =\int_{a}^{b}\left|T_{1}(s, p, w(p))-T_{2}(s, p, z(p))\right|^{2} d p+2 \\
& \leq \int_{a}^{b}\left(\frac{\varphi_{c b}(w, z)}{(b-a) e^{t}}-\frac{2}{b-a}\right) d p+2 \\
& =\frac{\varphi_{c b}(w, z)}{e^{t}}=\lambda_{1} \varphi_{c b}(w, z) \\
& =\lambda_{1} \varphi_{c b}(w, z)+\lambda_{2} \frac{\varphi_{c b}(w, \mathfrak{S} w) \varphi_{c b}(z, \mathfrak{T} z)}{1+\varphi_{c b}(w, \mathfrak{T} z)+\varphi_{c b}(w, z)} .
\end{aligned}
$$

Hence, all the conditions of Theorem 5 are satisfied for $\mathrm{a}_{1}+s \mathrm{a}_{2}(=0)<1$, with $t>0$. Therefore, the system of integral equations (44) and (45) has a unique common solution.

\section{Conclusion}

In this paper, we proved some common fixed point theorems for rational contraction mapping on complex partial $b$-metric space. An illustrative example and application on complex partial $b$-metric space is given.

\section{Data Availability}

No data were used to support the study.

\section{Conflicts of Interest}

The authors declare that they have no conflicts of interest.

\section{Authors' Contributions}

All authors contributed equally to the writing of this paper. All authors read and approved the final manuscript.

\section{Acknowledgments}

$\forall$ The ${ }^{0}$ fourth-named author extends appreciation to the Deanship of Scientific Research at King Khalid University for funding work through research groups program under grant R.G.P.1/15/42.

\section{References}

[1] I. A. Bakhtin, "The contraction mappings principle in quasimetric spaces," Functional Analysis, vol. 30, pp. 26-37, 1989.

[2] S. Czerwick, "Contraction mappings in b-metric spaces," Acta Mathematica et Informatica Universitatis Ostraviensis, vol. 1, pp. 5-11, 1993.

[3] A. Azam, B. Fisher, and M. Khan, "Common fixed point theorems in complex valued metric spaces," Numerical Functional Analysis and Optimization, vol. 32, no. 3, pp. 243-253, 2011.

[4] K. P. R. Rao, P. R. Swamy, and J. R. Prasad, "A common fixed point theorem in complex valued b-metric spaces," Bulletin of Mathematics and Statistics Research, vol. 1, no. 1, 2013.

[5] P. Dhivya and M. Marudai, "Common fixed point theorems for mappings satisfying a contractive condition of rational expression on a ordered complex partial metric space," Cogent Mathematics, vol. 4, no. 1, p. 1389622, 2017.

[6] M. Gunaseelan, "Generalized fixed point theorems on complex partial b-metric space," International Journal of Research and Analytical Reviews, vol. 6, no. 2, 2019.

[7] G. Mani, A. J. Gnanaprakasam, Y. Li, and Z. Gu, "The existence and uniquenes solution of nonlinear integral equations via common fixed point theorems," Mathematics, vol. 9, no. 11, p. 1179, 2021.

[8] T. G. Bhaskar and V. Lakshmikantham, "Fixed point theorems in partially ordered metric spaces and applications," Nonlinear Analysis: Theory, Methods and Applications, vol. 65, no. 7, pp. 1379-1393, 2006.

[9] M. Gunaseelan and L. N. Mishra, "Coupled fixed point theorems on complex partial metric space using different type of contractive conditions," Scientific Publications of the State University of Novi Pazar Series A: Applied Mathematics, Informatics and mechanics, vol. 11, no. 2, pp. 117-123, 2019.

[10] A. Latif, T. Nazir, and M. Abbas, "Stability of fixed points in generalized metric spaces," Journal of Nonlinear and Variational Analysis, vol. 2, no. 3, pp. 287-294, 2018.

[11] M. Abbas, I. Beg, and B. T. Leyew, "Common fixed points of $(\mathrm{R}, \alpha)$-generalized rational multivalued contractions in R-complete b-metric spaces," Communications in Optimization theory, vol. 2019, p. 14, 2019.

[12] G. Mani, L. N. Mishra, V. N. Mishra, I. A. Baloch, and M. de la Sen, "Application to coupled fixed-point theorems on complex partial b-metric space," Journal of mathematics, vol. 2020, Article ID 8881859, 11 pages, 2020.

[13] A. Leema Maria Prakasam and M. Gunaseelan, "Common fixed point theorems using (CLR) and (E.A.) properties in complex partial $b$-metric space," Advances in Mathematics: Scientific Journal, vol. 9, no. 5, pp. 2773-2790, 2020. 
[14] F. Gu and W. Shatanawi, "Some new results on common coupled fixed points of two hybrid pairs of mappings in partial metric spaces," Journal of Nonlinear Functional Analysis, vol. 2019, no. 1, 2019.

[15] D. Baleanu, S. Rezapour, and H. Mohammadi, "Some existence results on nonlinear fractional differential equations," Philosophical Transactions of the Royal Society A: Mathematical, Physical and Engineering Sciences, vol. 371, no. 1990, 2013.

[16] W. Sudsutad and J. Tariboon, "Boundary value problems for fractional differential equations with three-point fractional integral boundary conditions," Advances in Difference Equations, vol. 2012, no. 1, 2012.

[17] M. S. Aslam, M. F. Bota, M. S. R. Chowdhury, L. Guran, and N. Saleem, "Common fixed points technique for existence of a solution of Urysohn type integral equations system in complex valued b-metric spaces," Mathematics, vol. 9, no. 4, p. 400, 2021.

[18] G. Mani, A. J. Gnanaprakasam, R. Kalaichelvan, and Y. U. Gaba, "Results on complex partial b-metric space with an application," Mathematical Problems in Engineering, vol. 2021, Article ID 5565470, 10 pages, 2021.

[19] M. I. Abbas and M. Alessandra Ragusa, "Solvability of Langevin equations with two Hadamard fractional derivatives via Mittag-Leffler functions,” Applicable Analysis, pp. 1-15, 2021.

[20] L. Chen, S. Huang, C. Li, and Y. Zhao, "Several fixed-point theorems for -contractions in complete Branciari -metric spaces and applications," Journal of Function Spaces, vol. 2020, Article ID 7963242, 10 pages, 2020.

[21] M. I. Abbas and M. A. Ragusa, "On the hybrid fractional differential equations with fractional proportional derivatives of a function with respect to a certain function," Symmetry, vol. 13, no. 2, p. 264, 2021.

[22] Z. Huang, D. Zhao, and H. Li, "Boundary Schwarz lemma and rigidity property for holomorphic mappings of the unit polydisc in C-n," Univerzitet u Nišu, vol. 34, no. 9, pp. 2813$2818,2020$.

[23] F. Rouzkard, "Common fixed point theorems for two pairs of self-mappings in complex-valued metric spaces," Eurasian Mathematical Journal, vol. 10, no. 2, pp. 75-83, 2019. 